\title{
Analyse der Hauptbehandllungskodierung der medizinischen Statistik für orthopädische Behandlungen mittels Spitaleinzugsgebieten (small area analysis)
}

\author{
Klazien Matter-Walstra, \\ Marcel Widmer, \\ Franziska Schoeni-Affolter, \\ Cornelia Balmer, \\ Andre Busato
}

Interessenverbindungen: keine.

Korrespondenz:

PD Dr. med. A. Busato

Universität Bern

Medizinische Fakultät

MEM Center, IEFO Institut für

evaluative Forschung in

der orthopädischen Chirurgie

Stauffacherstrasse 78

CH-3014 Bern

Tel. 0316315962

Fax 0316315961

andre.busato@MEMcenter.unibe.ch

\section{Zusammenfassung}

Small-Area-Analysen der Spitalnutzung in der Schweiz bedingen eine landesweite einheitliche Erfassung der erbrachten medizinischen Leistungen und eine Vollständigkeit der Diagnose- und Behandlungskodierung. Untersucht wird, ob die Kodierung der Hauptbehandlung von orthopädischen Fällen in der Schweiz regionale Unterschiede aufweist und welche Konsequenzen für Small-Area-Analysen der Schweiz sich daraus ergeben.

Die Schweiz kann für die Orthopädie in 69 Spitaleinzugsgebiete eingeteilt werden. Analysiert wurde die Behandlungskodierung von 216550 orthopädischen Fällen (2002-2003). Insgesamt sind 85,2\% dieser Fälle mit einem Hauptbehandlungskode versehen. Es wird gezeigt, dass die Wahrscheinlichkeit einer Kodierung von Alter, Geschlecht, Ort der Behandlung (Wohnbzw. «Fremd»-Spitaleinzugsgebiet) sowie von der Region, in der die Hospitalisation stattfindet, abhängig ist. Diese regionalen Differenzen werden vermutlich durch eine verschiedene Auslegung der Kodierungsrichtlinien oder durch unterschiedliche finanzielle Anreizsysteme verursacht. Die Resultate der Small-Area-Analysen können dadurch beeinträchtigt werden.

\section{Einleitung}

Seit Januar 1997 werden alle Schweizer Spitalentlassungen jährlich beim Bundesamt für Statistik in der «Medizinischen Statistik der stationären Einrichtungen» erfasst. Jeder dieser Fälle besitzt einen Wohnsitzhinweis in Form eines Codes, der eine oder mehrere Postleitzahlen (PLZ) zusammenfasst. Diese neu definierten Gebiete werden «MedStat-Regionen» genannt. Die Schweiz wird in 612 MedStat-Regionen unterteilt, von denen 240 mindestens 1 Spital beherbergen. Jede Region hat zwischen 3500 und 10000 Einwohnern und wird nach sozioökonomischen und geographischen Kohärenzkriterien gebildet, deshalb sind diese Regionen einheitlich und vergleichbar [1]. Nach einer Anlaufzeit darf die Datenlage der medizinischen Statistik ab 2002 als vollständig betrachtet werden. Mittels dieser Statistik kann eine exakte Bestandsaufnahme des Versorgungszustandes und der Spitalnutzung der Schweizer Bevölkerung erstellt werden. Dazu eignet sich ausser den traditionell benutzten kantonalen Analysen auch die Untersuchung von sogenannten Spitaleinzugsgebieten. Spitaleinzugsgebiete sind Aggregate von MedStat-Regionen, die aus mindestens einer MedStat-Region und einem Spital bestehen. Die Bildung dieser Gebiete beruht auf der Methode der «small area analysis» [2-4]. Einzugsgebiete widerspiegeln die effektive Nutzung stationärer Leistungen durch die Bevölkerung im Zeitraum der Datenerhebung [5-9]. Diese Einzugsgebiete erlauben verschiedenste Aussagen wie z.B. über den Umfang ausserregionaler Behandlungen, Einblick in die geographische Struktur/Verteilung der Spitalnutzung oder über Schwankungen der Nutzung von Ressourcen und Patientenströme. Da die Aufteilung in Einzugsbiete sehr detaillierte Angaben ermöglicht, können exaktere Untersuchungen von Versorgungsengpässen und/oder Kapazitätsüberschüssen durchgeführt werden, als dies aufgrund kantonaler Grenzen möglich ist. Analysen können weiter verfeinert werden, indem Leistungsgruppen (Innere Medizin, Chirurgie), Diagnoseoder Behandlungsgruppen, Liegeklassen oder Spitaltypen (Akutspital, Rehabilitation) getrennt untersucht werden. Die Voraussetzung solcher Analysen ist allerdings eine landesweite einheitliche Erfassung der erbrachten medizinischen Leistungen und eine vollständige Kodierung der Diagnosen und Behandlungen [10]. Laut Kodierungshandbuch [11] müssen alle operativen sowie alle Behandlungen der roten Liste [12] zwingend mit mindestens einem Hauptbehandlungskode (CHOP [13]) und diagnostische und therapeutische Massnahmen wenn möglich mit einem CHOP-Kode aus dem Kapitel 16, wenn dieser für die Beschreibung des Falles von Bedeutung ist, kodiert werden. Im weiteren ist die Kodierung der Hauptbehandlung, falls ein ent- 
sprechender CHOP-Kode vorhanden ist, für alle Fälle obligatorisch [14]. Nicht eingetragene CHOP-Angaben werden durch das Bundesamt für Statistik mit dem Kode «0000» ergänzt und könnten, je nach Handhabung der Kodierungsrichtlinien, folgendes bedeuten:

- die durchgeführte Behandlung gehört nicht $\mathrm{zu}$ den zwingend $\mathrm{zu}$ kodierenden CHOPKategorien [11];

- es handelt sich um eine diagnostische oder therapeutische Massnahme mit einem CHOPKode aus dem Kapitel 16, deren Eintrag als nicht bedeutend für die Beschreibung des Falles erachtet wird [11];

- für die durchgeführte Behandlung gibt es keinen CHOP-Kode;

- es wurde keine Behandlung durchgeführt;

- $\quad$ es wurde nicht kodiert (Missing).

Fehlende Behandlungs-(CHOP-) und/oder Diagnoseangaben (ICD-10 [15]) können zu Verzerrungen bei der Bewertung der Inanspruchnahme medizinischer Leistungen führen und SmallArea-Analysen erschweren. In der vorliegenden Arbeit wird die Bildung von Spitaleinzugsgebieten beschrieben, die mittels Entlassungsdaten für die Jahre 2002-2003 [16] gebildet wurden. Im Rahmen des NF-Projektes «Musculoskeletal Health - Chronic Pain» wird für orthopädische Fälle (alle Fälle mit einer Hauptdiagnose «Krankheiten des Muskel-Skelett-Systems und des Bindegewebes» [M00-M99]) das Vorhandensein eines Hauptbehandlungskodes analysiert. Ziel dieser Arbeit ist die Untersuchung der Effekte individueller Faktoren sowie von regionalen Unterschieden in der Kodierungspraxis der Hauptbehandlungskodes. Die daraus resultierenden Konsequenzen für Small-Area-Analysen der Schweiz werden anschliessend diskutiert.

\section{Patienten und Methode}

\section{Bildung von Spitaleinzugsgebieten}

Die Bildung von Spitaleinzugsgebieten (HSA = Hospital Service Area) basiert auf allen Spitalentlassungen der Jahre 2002 und 2003 für Personen ab 15 Jahre. Der verwendete Datensatz (BfS) beinhaltet keine Daten von Spezialkliniken oder Rehabilitationskliniken.

Für die Bildung von Spitaleinzugsgebieten muss von jeder Spitalentlassung die Wohn-MedStat als auch die Behandlungs-MedStat bekannt sein. Die Bildung der Spitaleinzugsgebiete folgt in mehreren Schritten [3, 4, 17, 18]: Für jede MedStat wird berechnet, in welcher MedStat Fälle am häufigsten, zweit- oder dritthäufigsten behandelt werden. Für MedStat-Regionen, in denen ein Spital vorhanden ist, kann Behandlungs- und Wohn-MedStat die gleiche MedStat sein. Alle MedStats werden nun ihren häufigsten Behandlungs-MedStats zugewiesen. Theoretisch können so 240 primäre HSAs entstehen, da in der Schweiz mindestens 240 MedStats nur ein Spital haben.

Der zweite Schritt erfolgt gemäss der Pluralitätsregel. Diese besagt, dass, wenn die Summe der zweit- und dritthäufigsten Entlassungen höher ist als die häufigste und diese beiden MedStats zu einem anderen primären HSA gehören, die MedStats ebenfalls diesem primären HSA zugeordnet werden.

Weiter wird untersucht, ob die HSAs genügend Einwohner haben. HSAs mit weniger als 10000 Einwohnern werden mit einem Nachbar-HSA fusioniert, in die die meisten «nichtlokalen» Entlassungen erfolgen.

In einem letzten Schritt werden HSAs, in denen weniger als 50\% aller Entlassungen in der eigenen (lokalen) HSA erfolgen, mit einer Nachbar-HSA fusioniert, in der die meisten «nichtlokalen» Entlassungen stattfinden.

\section{Variablen}

Nach Bildung der HSA werden alle Entlassungen ihrer zugehörigen Wohn- und Behandlungs-HSA zugewiesen. Auf einzelne Entlassungsebenen werden folgende Variablen verwendet: Alter und Geschlecht des Falls, Wohn- und BehandlungsHSA, behandelndes Spital (in Form eines anonymen «BUR»-Nummer), Liegedauer, Hauptdiagnosekode (vierstelliger ICD-10-Kode) und daraus resultierende Hauptkategorien (ein- oder zweistelliger ICD-10-Kode), Hauptbehandlungskode (CHOP) und daraus resultierend, ob eine Hauptbehandlungskode eingetragen wurde: ja $(\mathrm{CHOP} \neq \ll 0000 »)$ oder nein $(\mathrm{CHOP}=« 0000 »)$ sowie die Lokalisation der Behandlung (in der eigenen Wohn-HSA oder in einer «fremden» HSA). Im Datensatz sind Rehospitalisationen der einzelnen Patienten nicht ersichtlich, daher ist im Folgenden nur von Fällen und nicht von Patienten die Rede.

Auf HSA-Ebene resultiert daraus die Gesamtanzahl aller Fälle, die in einer HSA hospitalisiert bzw. entlassen werden, die Prozentanzahl Fälle mit eingetragenem Behandlungskode $(\mathrm{CHOP} \neq$ «0000»), das durchschnittliche Alter der Fälle, der Anteil Frauen unter den Fällen, der Prozentanteil Entlassungen von Fällen, die nicht in der jeweiligen HSA wohnen.

\section{Ein- und Ausschlusskriterien}

Für diese Studie werden alle stationär behandelten Fälle im Alter ab 15 Jahre mit der ICD-10- 
Hauptkategorie «M» (Krankheiten des MuskelSkelett-Systems und des Bindegewebes [M00M99]) berücksichtigt, mit einer Liegedauer von maximal 99 Tagen.

\section{Statistische Analysen}

\section{Deskriptive Analysen}

Mit dem Chi-Quadrat-Test wurde die Hypothese überprüft, ob es Unterschiede gibt bei der CHOPKode-Vergabe zwischen Männern und Frauen oder lokal (in der eigenen Wohn-HSA) und nicht lokal (in einer «Fremd-HSA») behandelten Fällen. Unterschiede im Alter und in der Liegedauer zwischen Fällen mit und ohne CHOP-Kode wurden mittels T-Tests analysiert.

\section{Analysen auf HSA-Ebene}

Ein logistisches Regressionsmodel wurde verwendet, um die Wahrscheinlichkeit zu bestimmen, dass in einer bestimmten HSA kein CHOP eingetragen wurde. Neben der HSA wurden die Lokalität der Hospitalisation (LOK) und das Geschlecht (SEX) als Klassifikationsvariablen sowie das Alter als kontinuierliche Variable als erklärende Faktoren in das Modell eingeschlossen. Das behandelnde Spital wurde im Modell als Cluster-Variable miteinbezogen, um so den Effekt korrelierter Beobachtungen auf Niveau Spital zu berücksichtigen. Modell: CHOP (EVENT $=$ «ohne CHOP») = HSA (Referenz $=$ «Bern») SEX $($ Referenz $=«$ Frau $»)$ LOK $($ Referenz $=«$ Lokal behan delt») Alter.

Ein lineares Modell wurde benutzt, um den auf HSA-Niveau aggregierten Prozentanteil Fälle mit CHOP-Kode zu untersuchen, um eine even- tuelle beobachtete Varianz zu erklären. Im Modell wurden das Durchschnittsalter der Fälle (MEAN AGE), Anteil Frauen unter den Fällen (ANT-F) und der Anteil nichtlokaler Fälle (ANTNL) berücksichtigt. Modell: «Prozentanteil Fälle mit CHOP-Kode» = MEAN AGE ANT-F ANT-NL.

Korrelationen zwischen kontinuierlichen Variablen wurden analysiert mit Spearman's rho.

Statistische Analysen wurden mit SAS 9.1 ${ }^{\circledR}$ (SAS Institute Inc., Cary, NC, USA) durchgeführt, die geographische Darstellung der Resultate mit ArcGis (ArcView8.2 ${ }^{\circledR}$, ESRI, Redlands CA, USA).

\section{Resultate}

\section{Deskriptive Analyse (Tab. 1)}

In den Jahren 2002-2003 erfolgten 216550 Spitalentlassungen mit einer Hauptdiagnose der Hauptkategorie «M». Bei gut $85 \%$ aller Fälle $(85,2 \%, 95 \%$ Confidence Interval $[\mathrm{CI}]=85,1-$ $85,4 \%$ ) wird von den Spitälern ein CHOP-Kode eingetragen, wobei Männer öfter als Frauen einen CHOP-Kode erhalten (Männer 86,8\%, Frauen $83,9 \%, p<0,001)$. Fälle, die nicht in der eigenen Wohn-HSA behandelt werden (nichtlokale Fälle), erhalten zudem häufiger einen CHOP-Kode als solche aus der «eigenen» HSA (nichtlokale Fälle 90,2\%, lokale Fälle 82,9\%, p <0,001). Ausserdem sind Fälle, die einen CHOP-Kode erhalten haben, durchschnittlich $4,2$ ( $\mathrm{p}<0,05)$ Jahre jünger und liegen durchschnittlich 4,0 $(\mathrm{p}<0,05)$ Tage kürzer im Spital als solche, die keinen CHOP-Kode erhalten haben. Dieser Trend ist gleichwohl für Männer als auch für Frauen zu beobachten. Der Anteil Fälle mit CHOP steigt von $83,9 \%(95 \% \mathrm{CI}=83,7-84,1 \%)$

Tabelle 1

Analyse der M-Diagnose-Fälle mit und ohne Hauptbehandlungskode.

\begin{tabular}{|c|c|c|c|c|}
\hline Geschlecht/Lokalität ${ }^{\circ}$ & CHOP & $N(\%)$ & Alter $(95 \% \mathrm{Cl})$ & Liegedauer in Tage $(95 \% \mathrm{Cl})$ \\
\hline Alle & Alle & 216550 & 57,43 & $9,4(8,0)$ \\
\hline \multirow[t]{2}{*}{ Alle } & Mit & $184602(85,2 \%)$ & $56,8(56,7-56,9)$ & $8,8(8,7-8,9)$ \\
\hline & Ohne & $31948(14,8 \%)$ & $61,0(60,8-61,2)^{*}$ & $12,8(12,7-12,9)^{*}$ \\
\hline Männer & Mit & $85244(86,8 \%)$ & $53,8(53,7-53,9)$ & $8,2(8,1-8,3)$ \\
\hline$(98153,45,3 \%)$ & Ohne & $12909(13,2 \%)$ & $55,9(55,6-56,2)^{*}$ & $11,5(11,4-11,7)^{*}$ \\
\hline Frauen & Mit & $99358(83,9 \%)$ & $59,4(59,3-59,5)$ & $9,4(9,3-9,5)$ \\
\hline$(118397,54,7 \%)$ & Ohne & $19039(16,1 \%)$ & $64,5(64,2-64,8)^{*}$ & $13,6(13,5-13,8)^{*}$ \\
\hline Lokal behandelt & Mit & $122562(82,9 \%)$ & $58,1(58,0-58,3)$ & $9,1(9,0-9,2)$ \\
\hline$(147766,68,2 \%)$ & Ohne & $25204(17,1 \%)$ & $62,7(62,4-62,9)^{*}$ & $13,2(13,0-13,3)^{*}$ \\
\hline Nicht lokal behandelt & Mit & $62040(90,2 \%)$ & $54,1(54,0-54,3)$ & $8,4(8,3-8,5)$ \\
\hline$(68784,31,8 \%)$ & Ohne & $6744(9,8 \%)$ & $54,8(54,5-55,3)^{*}$ & $11,3(11,0-11,5)^{*}$ \\
\hline
\end{tabular}


im Jahr 2002 auf 86,5\% (95\% CI = 86,3-86,7\%) im Jahr 2003.

Der Einfluss des Alters auf die Vergabe von CHOP-Kodes ist nicht linear über das ganze Altersspektrum der Fälle verteilt (Abb. 1). Bis zur Altersgruppe der 75- bis 79jährigen gibt es keine grossen Schwankungen, aber für die älteren Jahrgänge sinkt der Prozentanteil Fälle mit

\section{Abbildung 1}

Anzahl Fälle und \%-Anteil mit CHOP, nach Altersgruppe, Geschlecht und Lokalität der Behandlung. $\mathrm{l}=$ lokal behandelt, $\mathrm{nl}=$ nichtlokal behandelt

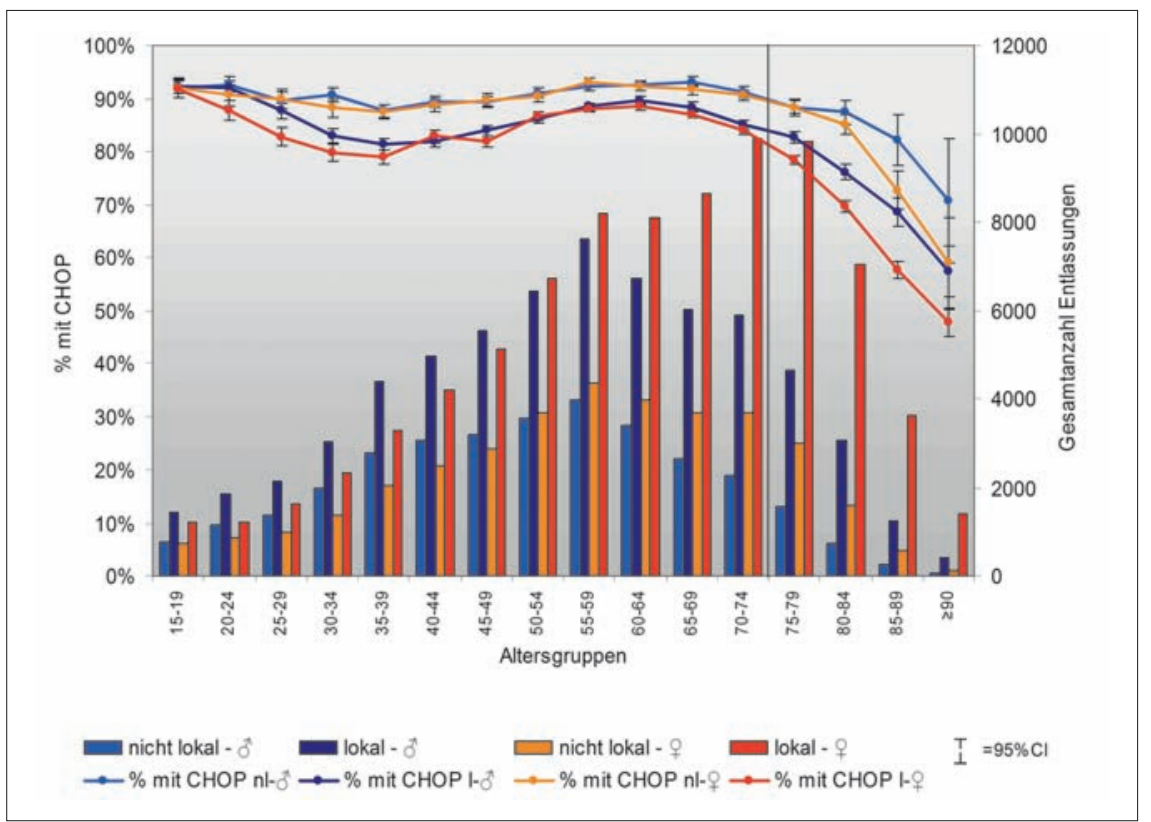

\section{Abbildung 2}

Durchschnittliche Liegedauer der einzelnen Altersgruppen mit und ohne Hauptbehandlungskode (korrigiert für Geschlecht und Lokalität der Hospitalisation).

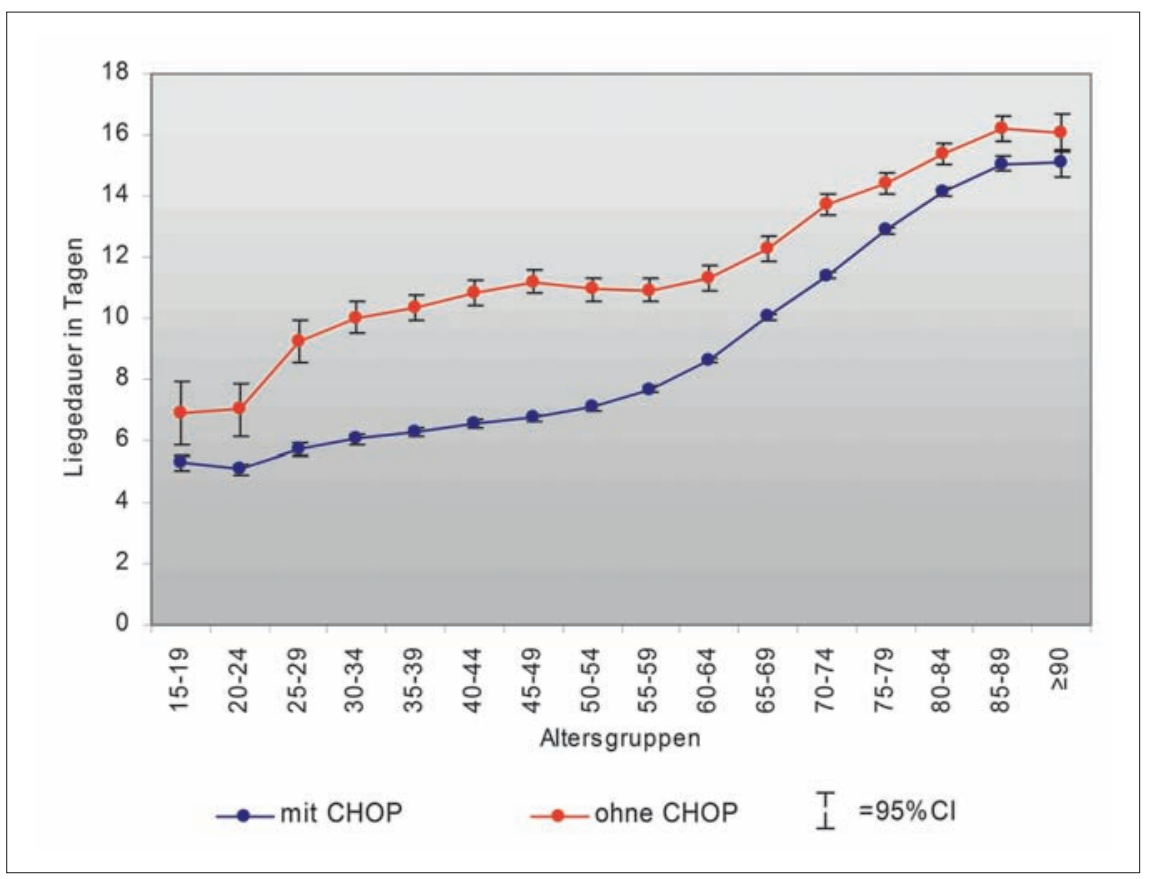

CHOP rapide $\mathrm{ab}$, und zwischen Frauen und Männer werden grössere Unterschiede bemerkbar. Die geographische Lokalisation der Behandlung zeigt über alle Altersgruppen und unabhängig vom Geschlecht einen konstanten Effekt. Die Liegedauer der Fälle nimmt mit dem Alter zu (Abb. 2, korrigiert für Geschlecht und Lokalisation der Hospitalisation), ist aber für alle Altersgruppen tiefer für Fälle mit CHOP.

\section{Analysen auf HSA-Ebene}

Aufgrund von 2361865 Entlassungen der Jahre 2002 und 2003 wurden 69 HSAs gebildet. Obwohl der Anteil «M»-Diagnose-Fälle, die mit eingetragenem CHOP-Kode erfolgen, landesweit mit $85,2 \%$ hoch erscheint, zeigen sich auf Niveau HSA grosse Unterschiede. Im Durchschnitt beträgt der Prozentanteil Fälle mit CHOP-Kode pro HSA $81,9 \%$, mit einem Maximum von $100 \%$ und einem Minimum von 33,1\%. Zweiundzwanzig Prozent $\left(\mathrm{r}^{2}=0,22\right)$ der beobachteten Varianz im Prozentanteil Entlassungen mit CHOP-Kode zwischen den HSAs kann im Model erklärt werden. Das Durchschnittalter der Fälle zeigt dabei einen signifikanten Effekt $(p<0,0024)$; Frauenanteil $(p=0,74)$ und der Anteil behandelten nichtlokalen (nicht aus der BehandlungsHSA stammenden) Fälle $(\mathrm{p}=0,89)$ nicht. Aufgrund der Beobachtung, dass das Alter erst ab 75 Jahre eine grössere Rolle bei der Vergabe von CHOP-Kodes zu spielen beginnt, wurde die gleiche Analyse nur für Fälle im Alter unter 75 Jahren durchgeführt. Für diese Population beträgt der Durchschnitt der Prozentanteil Fälle mit CHOP-Kode pro HSA 83,9\%, mit einem Maximum von $100 \%$ und einem Minimum von 39,5\%. Durchschnittsalter, Anteil nichtlokal Behandelter und Frauenanteil haben keinen signifikanten Einfluss. Geographisch bestehen signifikante Unterschiede zwischen den HSAs betreffend die Wahrscheinlichkeit (ODDS), in einem HSA keinen CHOP-Kode zu bekommen (Abb. 3), aber es lassen sich keine klaren Muster hinsichtlich der Prozentanteil Fälle mit CHOP pro HSA erkennen. Sowohl städtische als auch ländliche, französisch- oder deutschsprachige HSAs zeigen hohe oder tiefe Werte. Eine Ausnahme ist das Tessin, das für alle vier ihm zugehörigen HSAs tiefe Anteile von Fällen mit CHOP aufweist. Innerhalb einiger Kantone werden sowohl HSA, in denen viele Fälle mit CHOP-Kode, als auch solche, in denen nur wenige Fälle mit CHOP-Kode registriert werden, beobachtet. Des weiteren besteht keine Korrelation zwischen den Gesamtanzahl Entlassungen in einer HSA und der Anteil Entlassungen mit CHOP (Spearman-Korrelationskoeffizient $=0,10, p=0,41$ ). Sowohl HSAs 


\section{Abbildung 3}

ODDS «kein CHOP» pro HSA, Alter, Lokalität und Geschlecht korrigiert.

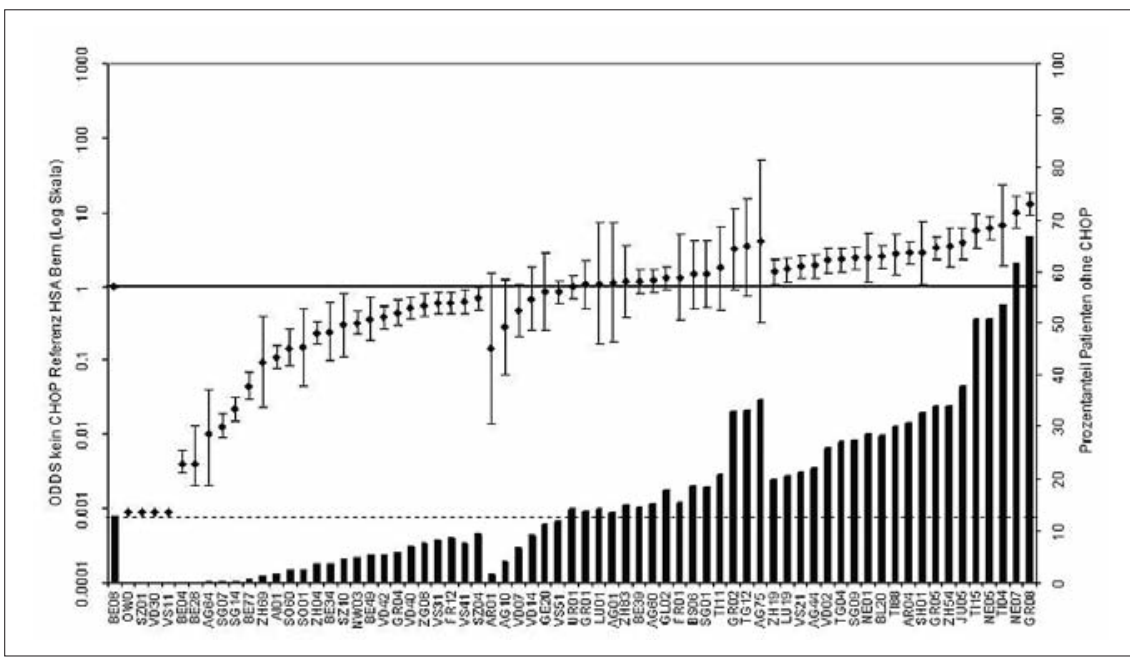

Abbildung 4

Verteilung der Anteil Fälle mit Hauptbehandlungskode über die HSA für verschiedene Diagnosen.

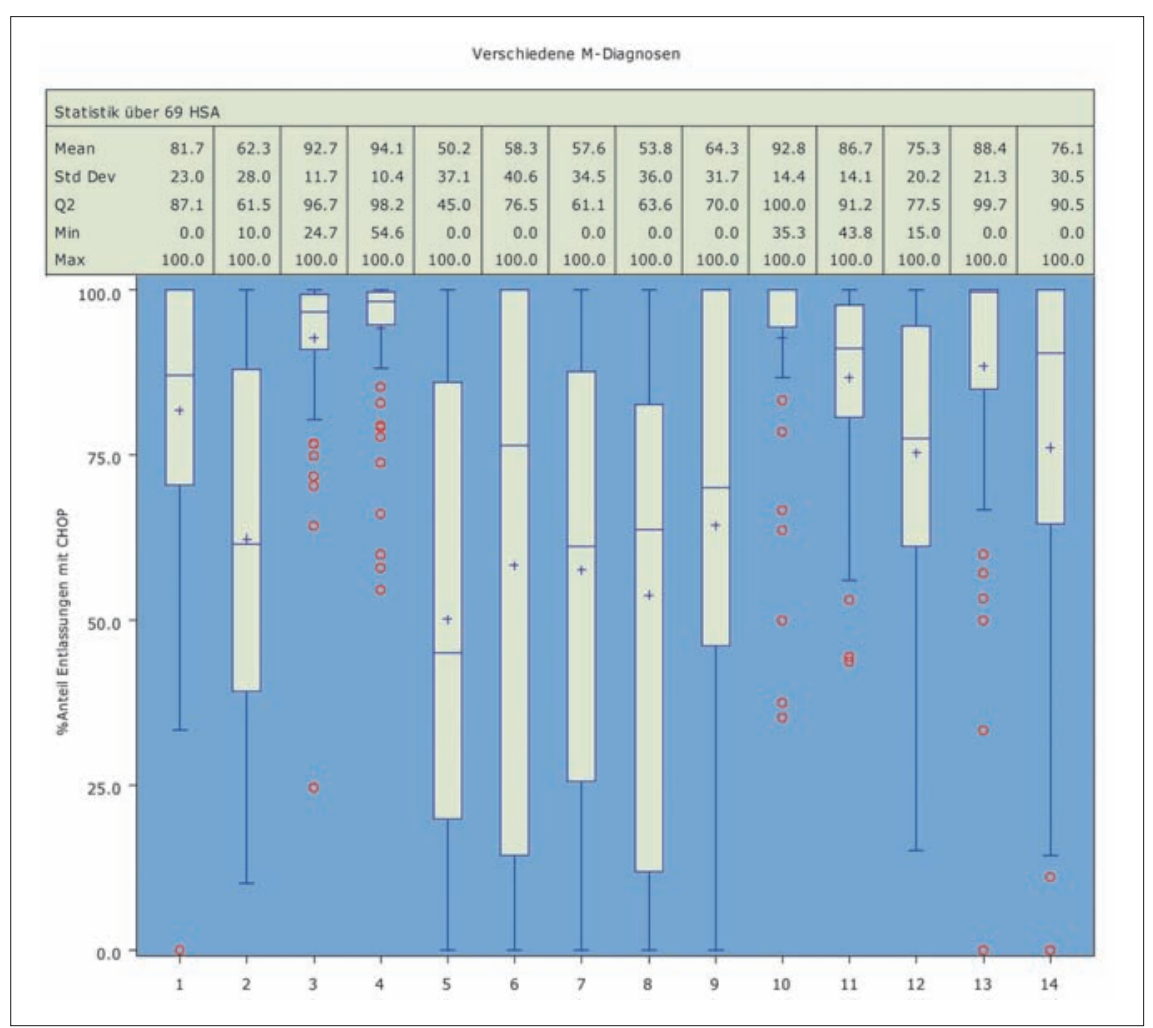

1 = infektiöse Arthropathien; 2 = entzündliche Polyarthropathien; 3 = Arthrosen; 4 = sonstige Gelenkserkrankungen; 5 = Bindegewebserkrankungen; 6 = Deformitäten Rücken/Wirbelsäule; 7 = Spondylopathien; 8 = sonstige Rücken/Wirbelsäule; 9 = Muskeln; 10 = Synovialis und Sehnen; 11 = sonstige Weichteilgewebeerkrankungen; 12 = Osteopathien; 13 = Chondropathien; 14 = sonstige M-Diagnosen.

Rotes $\mathrm{O}=$ Outliers (HSA mit einem Wert $>1,5^{\prime}$ Interquartilrange über den 75\%-Quartils); + und Q2 = Median und - Mean = Mittelwert; Boxbegrenzung = 25\%- und 75\%-Quartil. mit vielen als auch solche mit wenigen Fällen zeigen entweder hohe oder niedere Anteile mit CHOP.

Für verschiedene Diagnosen unterscheidet sich auf HSA-Niveau der durchschnittliche Prozentanteil Entlassungen mit CHOP-Kode stark. So haben Arthrosen durchschnittlich einen grossen Anteil Entlassungen mit CHOP-Kode (92,7\% mit $\mathrm{CHOP})$, während Bindegewebserkrankungen durchschnittlich pro HSA niedere Anteile Entlassungen mit CHOP aufweisen (durchschnittlich 50,2\%, Abb. 4). Die Streuung der Werte ist je nach Diagnose sehr unterschiedlich.

In vier HSAs haben $100 \%$ aller Fälle einen CHOP-Kode, in weiteren fünf dagegen fehlt bei über 50\% der Fälle der CHOP-Kode. Eine differenzierte Analyse der vergebenen CHOP-Kodes zeigt, dass in HSAs, in denen 100\% aller Entlassungen mit einem CHOP-Kode versehen sind, auch CHOP-Kodes, die nicht den operativen Behandlungen zugeordnet und damit «nicht zwingend zu kodieren sind», wie Injektionen, Rehabilitationsmassnahmen und Physiotherapie oder diagnostische Verfahren, vergeben werden. In HSAs, in denen über 50\% aller Entlassungen ohne CHOP-Kode sind, werden CHOP-Kodes solcher Art nicht registriert (Abb. 6).

\section{Diskussion}

Ende der sechziger Jahre erschienen erste Artikel, die über geographische Unterschiede in der Nutzung von medizinischen Einrichtungen berichteten $[9,19-24]$. In den folgenden Jahren wurden mehrere Hypothesen aufgestellt, um die beobachteten Varianzen von Nutzung, Nachfrage und Angebot zu erklären. Nebst soziodemographischen Faktoren der Patienten wurden hauptsächlich folgende Einflussgrössen untersucht: bestimmte Risikofaktoren in der Bevölkerung [23], Unter- und/oder Überversorgung [25], angebotinduzierte Nachfrage [26] oder Praxisstile $[27,28]$ (Präferenz für bestimmte Therapieformen). Die Methode der «small area analysis» wurde aber auch kritisiert, unter anderem wegen mangelnden oder fehlenden richtigen statistischen Verfahren oder ungenügender Datenqualität $[29,30]$.

Seit 1998 werden in der Schweiz alle Hospitalisationen registriert und stellen somit eine Vollerhebung der Spitalnutzung dar (Bundesamt für Statistik: 2003, 100\% aller Spitäler, $93 \%$ aller Fälle). Diese können als Grundlage für eine SmallArea-Analyse der Schweiz genutzt werden. Ziele solcher Analysen können, wie oben beschrieben, mehrfach sein, grundsätzlich sollten sie aber eine Unterstützung in der Spital- und Gesundheitsversorgungsplanung ermöglichen. 


\section{Abbildung 5}

Anteil der Fälle mit Hauptbehandlungskode und ODDS kein CHOP-Kode pro HSA (alle Fälle mit einer «M»-Hauptdiagnose).

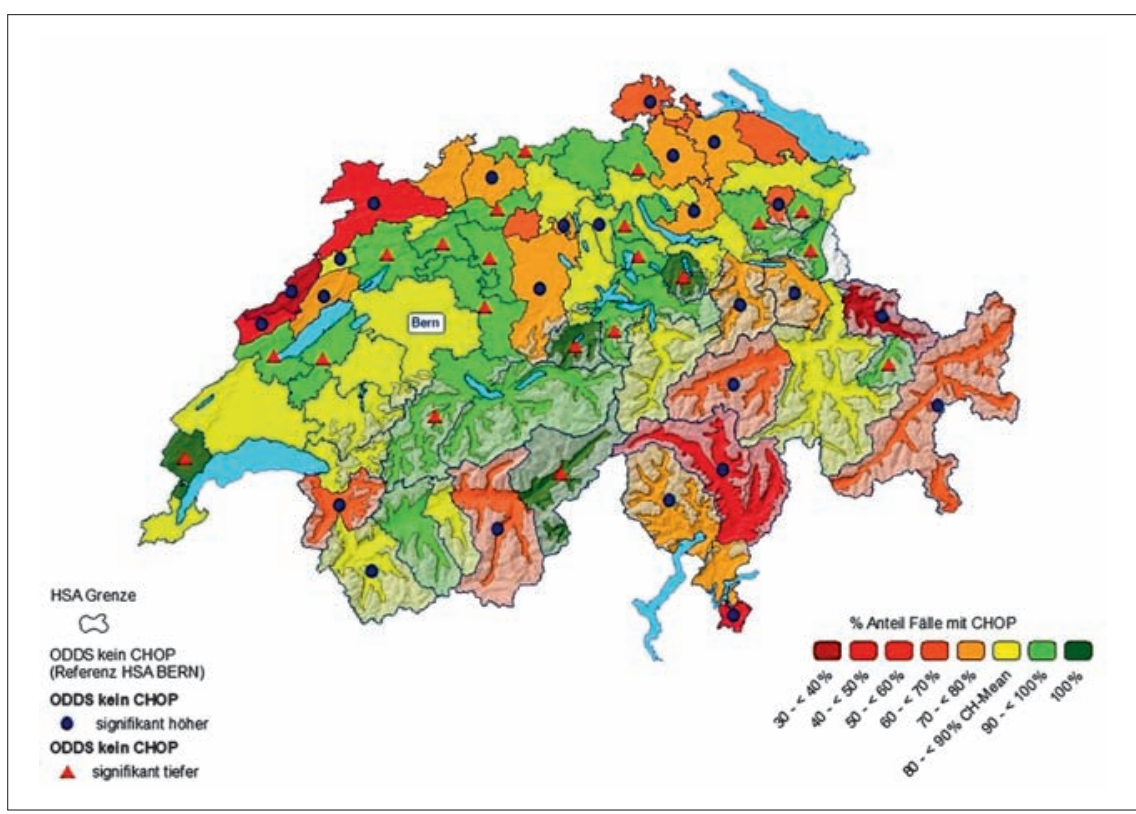

\section{Abbildung 6}

Hauptbehandlungskode in verschiedenen HSA-Typen (basierend auf den 20 häufigsten Hauptbehandlungskodes in HSAs, in denen 100\% aller Entlassungen mit einem CHOP-Kode erfolgen).

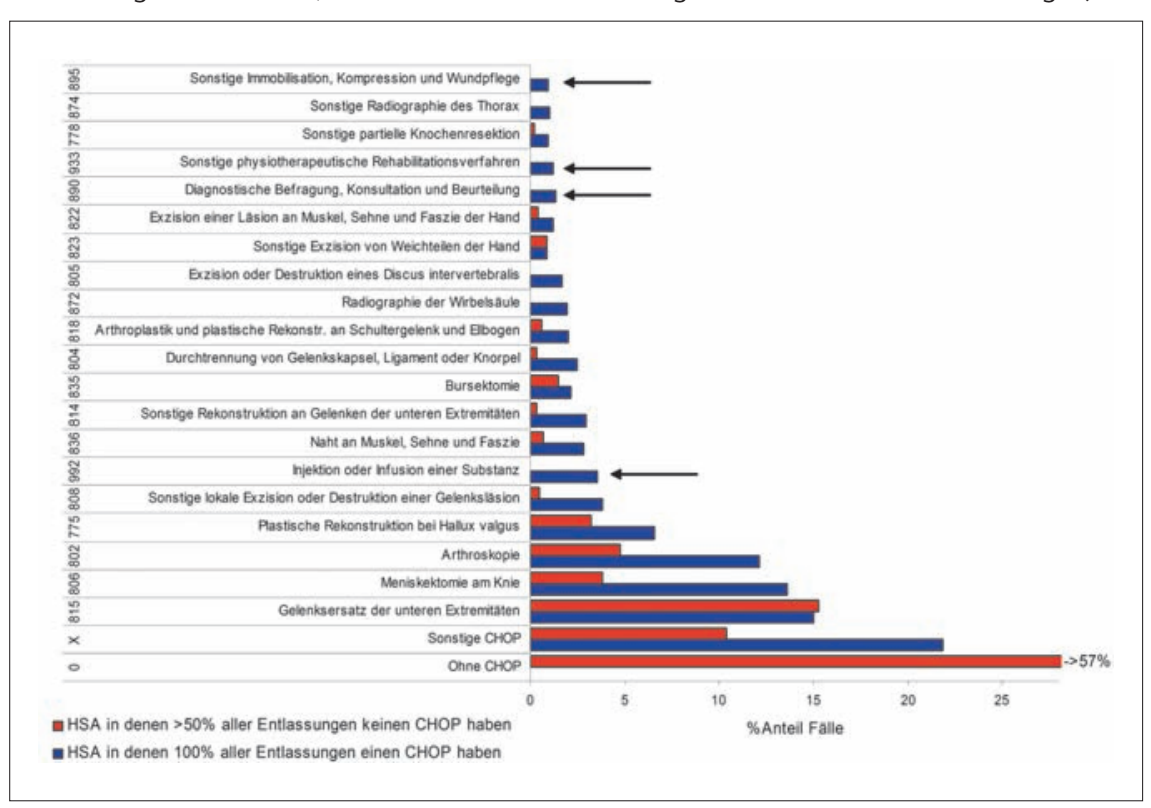

Voraussetzung für zuverlässige Analysen ist eine Vollständigkeit der Daten in bezug auf die gestellte(n) Diagnose(n) und durchgeführte(n) Behandlung(en). Für die Kodierung der Behandlungen sind die Anweisungen im Kodierungshandbuch [11] und weitere Verordnungen [14] zum Teil unschlüssig. Wie die einzelnen Spitäler diese Richtlinien handhaben, ist unbekannt, und folglich ist ein nicht eingetragener CHOPKode schwer zu interpretieren.
Eine uneinheitliche Kodierungspraxis kann erhebliche Folgen haben, wenn es um Fragestellungen in bezug auf die Spitalversorgung, -nutzung, Über-/Unterversorgung oder Praxisstile in der Schweiz geht.

Am Beispiel orthopädischer Entlassungen (d.h. alle Entlassungen mit einer Hauptdiagnose ICD-10 «M») lässt sich diese Problematik anschaulich darstellen. Insgesamt ist bei 85,2\% aller Entlassungen mit ICD-10 «M» (2002-2003) ein CHOP-Kode eingetragen, was im Vergleich zu den gesamten Entlassungen aller medizinischen Fachrichtungen in diesem Zeitraum mit etwa $73 \%$ der CHOP-kodierten Fälle eher hoch ist.

Die deskriptive Analyse der Fälle zeigt einige Auffälligkeiten. Im Alter ab etwa 70-74 Jahre nimmt der Anteil Fälle mit CHOP-Kode rapide ab, und dies bei Frauen stärker als bei Männern, obwohl die absolute Anzahl entlassener Frauen im Vergleich zu den Männern immer höher wird. Werden ältere Menschen und insbesondere Frauen eher weniger invasiv behandelt und bekommen deswegen keinen CHOP-Kode eingetragen, oder ändert sich das Krankheitsspektrum (im Alter und zwischen Männern und Frauen) und treten vermehrt Diagnosen auf, für deren Behandlung keine zwingende CHOP-Kodierung notwendig ist? Eine weitere Auffälligkeit ist, dass Fälle, die nicht lokal (nicht in der Wohn-HSA) hospitalisiert werden (unabhängig von Alter und Geschlecht), viel höhere Anteile mit einem CHOP-Kode aufweisen. Eine Erklärung für dieses Phänomen könnte sein, dass es sich bei diesen Fällen möglicherweise um operative Eingriffe handelt, die zwingend kodiert werden müssen und nicht in der eigenen Wohn-HSA durchgeführt werden können. Zudem ist denkbar, dass Fälle, die lokal hospitalisiert werden, eher diagnostische Abklärungs- oder Nachbetreuungshospitalisationen umfassen, in denen nicht eine eigentliche Behandlung durchgeführt wird. Die dritte Auffälligkeit ist, dass Fälle mit CHOP-Kode durchschnittlich viel kürzer hospitalisiert werden als solche ohne CHOP-Kode. Die grössten Unterschiede sind für die Altersgruppen der 25- bis 59jährigen zu beobachten. Es stellt sich die Frage, warum diese eher jungen Personen ohne CHOPKode so lange hospitalisiert sind. Was wird während ihres Spitalaufenthalts mit ihnen gemacht? Diese Frage lässt sich, ohne zu spekulieren, kaum beantworten.

Die geographische Verteilung (auf HSAEbene) der Prozentanteile Entlassungen mit CHOP zeigt ein zusätzlich schwierig zu interpretierendes Bild, mit HSAs, in denen 33\%, bis zu HSAs, in denen $100 \%$ der Entlassungen mit einer «M»-Diagnose mit CHOP-Kode erfolgen. 
Wenn nur Fälle der Altersgruppen der 15- bis 74jährigen analysiert werden, kann die beobachtete Varianz nicht mehr mit den untersuchten Eigenschaften der Patientenpopulation (Alter, Geschlecht oder Lokalität der Hospitalisation) erklärt werden. Zusätzlich sind je nach Untergruppe der «M»-Diagnosen die beobachteten Varianzen auf HSA-Ebene noch grösser (z. T. 0 bis zu 100\% der Entlassungen mit CHOP). Diesen Beobachtungen können sowohl medizinische wie auch politische/ökonomische Faktoren zugrunde liegen. Unter der Annahme, dass landesweit nur operative Behandlungen kodiert werden (CHOP der Kapitel 1-15 und rote Liste), sind verschiedene medizinische Erklärungen denkbar. Naheliegend wäre, dass in bestimmten HSAs keine oder nur wenige operative Eingriffe, die einen CHOP-Kode erzwingen, durchgeführt werden. Zudem ist es möglich, dass in diesen HSAs eher Fälle hospitalisiert werden, die Behandlungen bedingen, für die eine CHOP-Kodierung nicht zwingend notwendig ist. Eine dritte Erklärung könnte sein, dass in verschiedenen HSAs für bestimmte Erkrankungen andere nichtoperative Therapien angewandt werden als in anderen HSAs. Diese letzte Hypothese lässt sich nur schlecht überprüfen, solange unklar ist, welche Behandlung diejenigen Fälle bekommen haben, die ohne CHOP-Kode entlassen wurden.

In politischer und/oder ökonomischer Hinsicht könnten hohe CHOP-Kodierungsraten in bestimmten HSAs darauf hinweisen, dass in diesen HSAs entweder in bezug auf die Kodierung andere Richtlinien vorliegen und/oder es für die Spitäler in diesen HSAs finanziell von Vorteil ist, alle Entlassungen mit einem bestimmten CHOP zu kodieren. Als durchführende Organe sind die Kantone und Gemeinden zuständig für die Erhebung der medizinischen Statistikdaten. Je nach Kanton, aber auch je nach Spitaltyp dürfte die Finanzierung der Kodierung selber und/oder eine Kopplung der Fallentgeltung mit ihrer Kodierung unterschiedlich ausfallen, was entweder als Anreiz zum Kodieren [31] oder zum Kodierungsminimalismus führen könnte.

Die tatsächlichen Gründe für die beobachteten regionalen Unterschiede sind vermutlich in einer Mischung aus den oben genannten medizinischen und politisch-ökonomischen Erklärungen zu suchen und könnten für Small-Area-Analysen der Schweiz einige Konsequenzen haben:

- Analysen der geographischen Varianzen für bestimmte Operations-/Behandlungsraten können zu einer Unterbewertung in Regionen mit unvollständiger Kodierung führen;

- Analysen in bezug auf regional unterschiedliche Behandlungsverfahren können erschwert werden. Die Frage, ob in einigen Regionen z.B. Gelenkbeschwerden eher medikamentös oder mit Physiotherapie bzw. operativ behandelt werden, kann strenggenommen nur für Regionen mit vergleichbarer Kodierungspraxis beantwortet werden;

- Untersuchungen in bezug auf Über- oder Unterversorgung sowie Analysen betreffend «angebotinduzierte Nachfrage» können zu verzerrten Resultaten führen, wenn in einigen Regionen bestimmte Behandlungen kodiert werden und in anderen nicht, obwohl dort die gleichen Behandlungen durchgeführt werden;

- Varianzanalysen regionaler Liegedauerunterschiede bei bestimmten Diagnosen werden ungenau, wenn die durchgeführten Behandlungen nicht als erklärende Faktoren in die Berechnungen eingeschlossen werden können.

An dieser Studie kann kritisiert werden, dass die Analyse auf HSA-Ebene und nicht direkt pro Spital erfolgt. Aus Gründen des Datenschutzes dürfen keine Aussagen über einzelne Spitäler gemacht werden. Im weiteren ermöglicht die Bildung von HSAs eine sinnvolle Aggregation von (Spital-)Daten mit dem Vorteil, dass sogenannte «Marktgebiete» gebildet werden, die als Basis für statistische Verfahren verwendet werden können. Der Nachteil solcher aggregierter Auswertungen ist, dass in einer HSA ein Mix aus «vollständig» und «weniger vollständig» kodierenden Spitälern bestehen kann.

Fazit dieser Studie ist, dass Entlassungen mit einer Hauptdiagnose «Krankheiten des MuskelSkelett-Systems und des Bindegewebes» in den Jahren 2002-2003 in 85\% mit einem Hauptbehandlungskode versehen wurden, Tendenz steigend. Auf individueller Ebene haben Geschlecht, Alter und Lokalisation der Hospitalisation einen Einfluss auf die Wahrscheinlichkeit, dass ein Hauptbehandlungskode eingetragen wird. Auf HSA-Ebene spielt die Region, in der die Hospitalisation stattgefunden hat, eine zusätzliche Rolle dabei, ob für den Fall ein Hauptbehandlungskode eingetragen wird. Diese regionalen Unterschiede geben zu denken und müssen womöglich in unterschiedlichen Auslegungen der Kodierungsrichtlinien und oder in verschiedenen Anreizsystemen gesucht werden. Für Small-Area-Analysen der Spitalnutzung in der Schweiz können diese bestehenden regionalen Differenzen die Ergebnisse beeinträchtigen. Es besteht die Gefahr, dass regional unterschiedliche Behandlungsraten nicht aufgrund von Faktoren wie Verfügbarkeit und Inanspruchnahme 
entstehen, sondern eine unterschiedliche Qualität der Kodierung als Ursache haben. Die bevorstehende landesweite Einführung des Fallpauschalensystems SwissDRG (2009/2010) [32, 33] dürfte auf die Vollständigkeit der Erhebung der Hauptbehandlungsdaten eine positive Auswirkung haben.

\section{Danksagung}

Dieses Projekt wurde unterstützt durch das Nationalfonds Research Programm NFP 53 «Musculoskeletal Health - Chronic Pain» (Project 405340-104607).

\section{Literatur}

1 MicroGIS. MedStat Documentation. 2002. www.microgis.ch/d/medstat_d.

2 Goodman DC, Mick SS, Bott D, Stukel T, Chang CH, Marth N, et al. Primary care service areas: a new tool for the evaluation of primary care services. Health Serv Res. 2003;38:287-309.

3 Klauss G, Staub L, Widmer M, Busato A. Hospital service areas - a new tool for health care planning in Switzerland. BMC Health Serv Res. 2005;5:33.

4 Matter-Walstra K, Widmer M, Busato A. Analysis of patient flows for orthopedic procedures using small area analysis in Switzerland. BMC Health Serv Res. 2006;6:119.

5 Chassin MR, Brook RH, Park RE, Keesey J, Fink A, Kosecoff J, et al. Variations in the use of medical and surgical services by the Medicare population N Engl J Med. 1986;314:285-90.

6 Keller RB, Soule DN, Wennberg JE, Hanley DF. Dealing with geographic variations in the use of hospitals. The experience of the Maine Medical Assessment Foundation Orthopaedic Study Group. J Bone Joint Surg Am. 1990;72:1286-93.

7 Swart E, Wolff C, Deh S. Häufigkeit und kleinräumige Variabilität von Operationen. Chirurg. 2000;71:109-14

8 Veugelers PJ, Hornibrook S. Small area comparisons of health: applications for policy makers and challenges for researchers. Chronic Dis Can. 2002, 23:100-10

9 Wennberg J, Gittelsohn A. Small area variations in health care delivery. Science. 1973;182:1102-8.

10 Barazzoni F, Barrès A-M, Burnand B, Francioli $\mathrm{P}$, Gaspoz J-M, Guillain H, et al. Die Qualität der Gesundheitsversorgung in den schweizerischen Spitäler: Analyse von sechs Indikatoren. Anhang Kodierung der Diagnosen und Behandlungen. Lausanne: FoQual; 2000. www.foqual.ch $\rightarrow$ Archives.

11 Bundesamt für Statistik. Kodierungshandbuch. Neuenburg: BFS; 2002.

12 Bundesamt für Statistik. Rote Liste. Neuenburg: BFS; 2002.

13 Bundesamt für Statistik. Schweizerische Operationsklassifikation (CHOP), ICD-9-CM. Volume 3. Neuenburg: BFS; 2004.

14 Bundesamt für Statistik. Obligatorische Kodierung der Hauptbehandlung. Neuenburg: BFS; 2001.
15 Swiss federal statistic office. icd-10, cim-10 for Switzerland. www.icd10.ch.

16 Bundesamt für Statistik. Medizinische Statistik der Krankenhäuser. Neuenburg: BFS; 2007.

17 Goodman DC, Green GR. Assessment tools: small area analysis. Am J Med Qual. 1996;11:S12-4.

18 Rising WR. Generalizing the plurality method for forming hospital service areas. Clin Perform Qual Health Care. 1993;1:199-204.

19 Lewis CE. Variations in the incidence of surgery. N Engl J Med. 1969;81:880-4.

20 Wennberg JE, Gittelsohn A. Health care delivery in Maine I: patterns of use of common surgical procedures. J Maine Med Assoc. 1975;66:123-30, 149.

21 Wennberg JE, Gittelsohn AM. A small area approach to the analysis of health system performance. Hyattsville: U.S. Department of Health and Human Services, Public Health Service, Health Resources Administration, Bureau of Health Planning; 1980.

22 McPherson K, Wennberg JE, Hovind OB, Clifford P. Small-area variations in the use of common surgical procedures: an international comparison of New England, England, and Norway. N Engl J Med. 1982;307:1310-4.

23 Knickman JR, Foltz AM. Regional differences in hospital utilization. How much can be traced to population differences? Med Care. 1984;22:971-86.

24 Paul-Shaheen P, Clark JD, Williams D. Small area analysis: a review and analysis of the North American literature. J Health Polit Policy Law. 1987;12:741-809.

25 Wennberg J, Freeman JL, Culp WJ. Are hospital services rationed in New Haven and over-utilized in Boston? Lancet. 1987;1:1185-9.

26 Richardson JR, Peacock SJ. Supplier-induced demand: reconsidering the theories and new Australian evidence. Appl Health Econ Health Policy. 2006;5:87-98.

27 Pekoz EA, Shwartz M, Iezzoni LI, Ash AS, Posner MA Restuccia JD. Comparing the importance of disease rate versus practice style variations in explaining differences in small area hospitalization rates for two respiratory conditions. Stat Med. 2003;22: 1775-86.

28 Stano M. Evaluating the policy role of the small area variations and physician practice style hypotheses. Health Policy. 1993;24:9-17.

29 Stano M. Further issues in small area variations analysis. J Health Polit Policy Law. 1991;16:573-88.

30 Volinn E, Diehr P, Ciol MA, Loeser JD. Why does geographic variation in health care practices matter? (And seven questions to ask in evaluating studies on geographic variation). Spine. 1994; 19:2092S-2100S.

31 Zehnder R. Fehlanreize sind zu vermeiden. Newsletter SwissDRG. 2004;(2):4.

32 Swinarski Huber Z. Les aléas de l'introduction de la classification DRG. Rev Med Suisse. 2006;2:2002, 2004-5.

33 Fischer W. Verschiedene Artikel zu SwissDRG. www.swissdrg.org $\rightarrow$ Literatur. 